\title{
Effects of Regional Versus General Anesthesia on Outcomes After Total Hip Arthroplasty
}

\author{
A Retrospective Propensity-Matched Cohort Study \\ Mohammad A. Helwani, MD, Michael S. Avidan, MBBCh, Arbi Ben Abdallah, PhD, Dagmar J. Kaiser, MD, \\ John C. Clohisy, MD, Bruce L. Hall, MD, and Heiko A. Kaiser, MD \\ Investigation performed at the Washington University in St. Louis School of Medicine, St. Louis, Missouri
}

\begin{abstract}
Background: Many orthopaedic surgical procedures can be performed with either regional or general anesthesia. We hypothesized that total hip arthroplasty with regional anesthesia is associated with less postoperative morbidity and mortality than total hip arthroplasty with general anesthesia.

Methods: This retrospective propensity-matched cohort study utilizing the American College of Surgeons National Surgical Quality Improvement Program (ACS NSQIP) database included patients who had undergone total hip arthroplasty from 2007 through 2011. After matching, logistic regression was used to determine the association between the type of anesthesia and deep surgical site infections, hospital length of stay, thirty-day mortality, and cardiovascular and pulmonary complications.
\end{abstract}

Results: Of 12,929 surgical procedures, 5103 (39.5\%) were performed with regional anesthesia. The adjusted odds for deep surgical site infections were significantly lower in the regional anesthesia group than in the general anesthesia group (odds ratio $[\mathrm{OR}]=0.38 ; 95 \%$ confidence interval $[\mathrm{Cl}]=0.20$ to $0.72 ; \mathrm{p}<0.01$ ). The hospital length of stay (geometric mean) was decreased by $5 \%(95 \% \mathrm{Cl}=3 \%$ to $7 \% ; \mathrm{p}<0.001)$ with regional anesthesia, which translates to 0.17 day for each total hip arthroplasty. Regional anesthesia was also associated with a $27 \%$ decrease in the odds of prolonged hospitalization $(\mathrm{OR}=$ $0.73 ; 95 \% \mathrm{Cl}=0.68$ to $0.89 ; \mathrm{p}<0.001)$. The mortality rate was not significantly lower with regional anesthesia $(\mathrm{OR}=0.78$; $95 \% \mathrm{Cl}=0.43$ to $1.42 ; \mathrm{p}>0.05)$. The adjusted odds for cardiovascular complications ( $\mathrm{OR}=0.61 ; 95 \% \mathrm{Cl}=0.44$ to 0.85 ) and respiratory complications $(\mathrm{OR}=0.51 ; 95 \% \mathrm{Cl}=0.33$ to 0.81 ) were all lower in the regional anesthesia group.

Conclusions: Compared with general anesthesia, regional anesthesia for total hip arthroplasty was associated with a reduction in deep surgical site infection rates, hospital length of stay, and rates of postoperative cardiovascular and pulmonary complications. These findings could have an important medical and economic impact on health-care practice.

Level of Evidence: Therapeutic Level III. See Instructions for Authors for a complete description of levels of evidence.

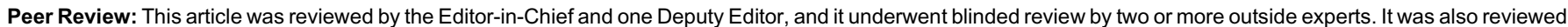

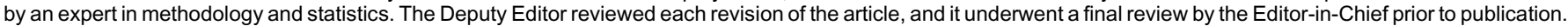
Final corrections and clarifications occurred during one or more exchanges between the author(s) and copyeditors.

$\mathrm{T}$ otal hip arthroplasty is a common and effective treatment for end-stage hip osteoarthritis. More than 200,000 primary total hip replacements and 36,000 revisions are performed each year in the United States'.
Complications following total hip arthroplasty are rare, with the rate of any major thirty-day complication commonly reported to be $0.2 \%$ to $1.2 \%$. Studies have suggested that older age and a worse American Society of Anesthesiologists (ASA)

Disclosure: None of the authors received payments or services, either directly or indirectly (i.e., via his or her institution), from a third party in support of any aspect of this work. One or more of the authors, or his or her institution, has had a financial relationship, in the thirty-six months prior to submission of this work, with an entity in the biomedical arena that could be perceived to influence or have the potential to influence what is written in this work. Also, one or more of the authors has had another relationship, or has engaged in another activity, that could be perceived to influence or have the potential to influence what is written in this work. The complete Disclosures of Potential Conflicts of Interest submitted by authors are always provided with the online version of the article. 
physical status classification ${ }^{2}$ as well as cardiovascular disease, pulmonary disease, and diabetes are associated with higher risks of death and postoperative complications $s^{3-5}$. Total hip arthroplasty is performed either with regional anesthesia, typically spinal anesthesia, or with general anesthesia, depending on the discretion of the anesthesiology team, surgeon preference, and patient choice. There is a lack of robust evidence regarding postoperative outcomes to inform the choice of anesthetic technique. Regional anesthesia has been hypothesized to decrease postoperative complications by reducing sympathetic activation and inflammation, by preventing venous stasis, and by avoiding tracheal intubation and positive pressure ventilation ${ }^{6-8}$. However, to our knowledge, the influence of anesthesia type on clinical outcomes has not been studied with large clinical trials, and the investigations that have been done have produced conflicting results ${ }^{9-11}$. In 2006, Mauermann et al. performed a meta-analysis of studies carried out from 1966 to 2005 and found lower rates of deep vein thrombosis and pulmonary embolism following total hip arthroplasties done with regional anesthesia compared with those done with general anesthesia ${ }^{12}$. They found no difference in transfusion requirements between the groups ${ }^{12}$, whereas other studies showed blood transfusion requirements to be lower with regional anesthesia ${ }^{13}$. Most of these positive studies were conducted years ago and may not reflect current practice. Over the last two decades, anesthesia care, surgical techniques, and postoperative care have improved, resulting in decreased perioperative morbidity and mortality despite increasing patient age and comorbidities ${ }^{14,15}$. While several recent studies utilizing large databases have suggested that regional anesthesia is superior for hip fracture surgical procedures, we are aware of only two studies of elective total hip arthroplasty that suggested that regional anesthesia (spinal or epidural) might be associated with lower rates of in-hospital complications and ninety-day mortality ${ }^{5,16}$.

Minimizing complications of total hip arthroplasty is an important public health concern. Despite the mounting evidence that regional anesthesia might be associated with improved outcomes, current American College of Surgeons National Surgical Quality Improvement Program (ACS NSQIP) data show that general anesthesia is still chosen for the majority of total hip arthroplasties. We therefore conducted a propensitymatched analysis to test the hypothesis that use of regional anesthesia decreases deep surgical site infection rates, hospital length of stay, postoperative mortality, and rates of cardiovascular and respiratory complications compared with general anesthesia in patients treated with total hip arthroplasty.

\section{Materials and Methods}

The ACS NSQIP is conducted under institutional review board approval at 1 Barnes-Jewish Hospital, Washington University in St. Louis. The present study was performed with use of the preexisting and deidentified Participant Use Data File and thus was exempt from further review. The STROBE (Strengthening the Reporting of Observational Studies in Epidemiology) checklist for observational studies was used to guide the methods of this study and to structure this manuscript ${ }^{17}$.

We conducted a retrospective cohort study with prospectively collected data on adults undergoing elective total hip replacement from January 1, 2007, through December 31, 2011, who were part of the ACS NSQIP. The purpose of the ACS NSQIP is to provide risk-adjusted clinical outcome data to participating hospitals to drive effective quality improvement. The database consists of 135 perioperative variables and thirty-day postoperative morbidity and mortality outcomes for patients treated with non-cardiac surgery.

Dedicated data personnel collect, validate, and submit the data at each site after rigorous uniform training and examination. Per ACS NSQIP guidelines, all variables, including complications, have predefined definitions and specifications (http://site.acsnsqip.org). During the submission of data from each institution, user information prompts are implemented to ensure completeness, uniformity, and validity of the data. Data integrity measures, including site visits, conference calls, an annual meeting of participants, interrater reliability site visits, and auditing, are regularly performed to safeguard the reliability and validity of the system's data. Several studies have demonstrated the high quality and validity of the ACS NSQIP database ${ }^{18}$; the combined results of audits revealed an overall disagreement rate of approximately $1.8 \%$ for all variables.

At the end of the calendar year 2011,316 hospitals were included in the data set; $49 \%$ of them were classified as "academic/teaching" and $51 \%$, as "non-teaching." There were three types of participation in this study period: classic, targeted, and essential. Classic programs have the least missing data on baseline characteristics of interest, so they were included for the analysis in this study (see details below).

\section{Inclusion and Exclusion Criteria}

We queried the ACS NSQIP database using Current Procedural Terminology (CPT) codes for primary total hip replacement (CPT 27130) and conversions to or revisions of total hip arthroplasties (CPT 27132, 27134, 27137, and 27138). All patients who received any regional anesthesia (spinal or epidural) or general anesthesia for primary or revision total hip arthroplasty in the classic programs were included. Demographic data, comorbidities, medical history, and surgical characteristics, including the type of procedure performed, were obtained for all identified patients. Case exclusion criteria were cases from non-classic programs as those programs had not collected data on ten of the thirty-two baseline variables, an age under sixteen years, preoperative mechanical ventilation as regional anesthesia is not an option when mechanical ventilation is used, preoperative coma, and no report of the type of anesthesia or use of a type other than spinal, epidural, or general anesthesia (Fig. 1).

\section{Outcomes and Interpretation}

Outcomes of interest were deep surgical site infection within thirty days after surgery and hospital length of stay from the day of the surgery until discharge. Hospital length of stay was used as a continuous variable, and it was also used as a categorical variable-i.e., prolonged hospital length of stay (yes or no), defined as longer than the average plus two standard deviations (SD) of the mean. Furthermore, the mortality rate as well as the rates of cardiovascular and pulmonary complications within thirty days after surgery were assessed. Cardiovascular complications and pulmonary complications were composite outcomes of other ACS NSQIP-defined events. A cardiovascular complication was defined as the occurrence of myocardial infarction, stroke, deep venous thrombosis, or pulmonary embolism. A pulmonary complication was defined as the occurrence of pneumonia or respiratory failure requiring intubation as specified within ACS NSQIP.

\section{Statistical Analysis}

Demographic and clinical characteristics were compared between the regional and general anesthesia groups by using Pearson chi-square tests for all categorical variables. Possible selection bias was addressed by performing propensity score matching. The propensity score for each individual is defined as the probability of receiving the treatment (regional anesthesia) given the patient's baseline characteristics and comorbidities (Table I). This incorporated thirty-two variables, including demographic variables and comorbidities (some of the thirty-two variables were combined to obtain a total of twenty-five baseline variables used for matching).

Multiple matching models with different matching ratios and calipers were evaluated to select a matching model with the overall best balance and the 


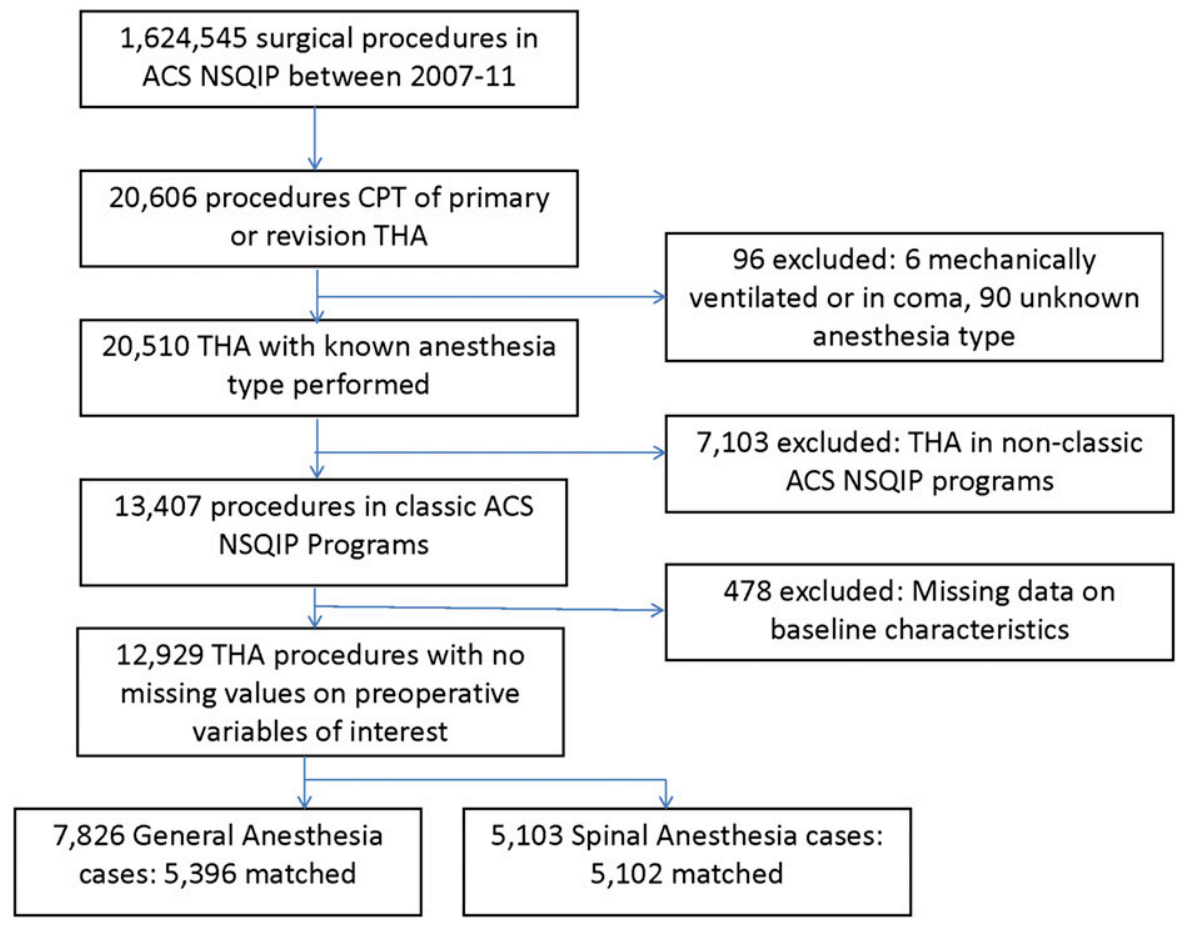

Fig. 1

Schematic demonstration of study sample. ACS NSQIP = American College of Surgeons National Surgical Quality Improvement Program, CPT = Current Procedural Terminology, and THA = total hip arthroplasty.

smallest reduction in the number of treated patients (regional anesthesia group). Nearest-neighbor matching with replacement with up to two controls (general anesthesia group) for each treated patient (regional anesthesia group) was used with a caliper of $0.05 \mathrm{SD}$ of a patient's predicted propensity score. Nearest-neighbor matching selects, for each treated individual $i$, the control individual with the smallest distance in propensity score from individual $i$. To account for the fact that some treated cases could be matched to only one control or one control could be used multiple times, weights were generated to correct for this disproportion. Every treated patient received a weight of one, and the weights for the matched control units were calculated by $n_{\text {treatedi }} /$ $n_{\text {controli }}$, where $n_{\text {treatedi }}$ and $n_{\text {controli }}$ were the number of treated and control units in stratum $i$. The control group weights were then scaled to sum to the number of uniquely matched control units. Following propensity score matching, the balance of the matched treated and control groups was evaluated by calculating variance ratios and absolute values of the standardized differences in means as well as by performing the Hotelling test, which is a generalization of the Student $\mathrm{t}$ statistic used in multivariate hypothesis testing ${ }^{19-21}$.

Unadjusted analyses were conducted for all outcomes before and after propensity score matching. To depict the influence of propensity score matching on the differences in outcomes, the $t$ test and chi-square test were used before matching; the weighted $t$ test and chi-square test were used after matching as deemed appropriate. Hospital length of stay was analyzed as a continuous outcome and as a binary outcome as prolonged hospital length of stay (a stay longer than the geometric mean $+[2 \times \mathrm{SD}])$. Because hospital length of stay was not normally distributed but rather was skewed to the right, a lognormal transformation was conducted to create a more normal distribution. Geometric means with standard deviations were calculated for both groups. Comparisons were conducted by using a weighted $t$ test on the lognormal transformed length of stay, and the geometric mean ratio was examined as an effect size estimate ${ }^{22,23}$. Prolonged hospital length of stay was analyzed by performing a chi-square test before matching and a weighted chi-square test after matching.

To further evaluate the potential effects of anesthesia type on outcome variables, multivariable analyses were conducted after propensity matching to adjust for specific covariates. Those covariates were defined a priori on the basis of clinical relevance reported in previous studies ${ }^{3,24}$. The number of covariates per logistic regression model was restricted to at least ten events per covariate to decrease the likelihood of overfitting.

As an age of greater than ninety years was not reported as a continuous variable in the ACS NSQIP data, we categorized age into three groups with comparable sizes.

Conditional multivariable logistic regression was used to evaluate the association between the type of anesthesia and deep surgical site infection, thirty-day mortality, and cardiovascular and pulmonary complications by using data from the matched patient cohort. A proportional hazard Cox regression analysis was performed to determine whether regional or general anesthesia had a significant influence on hospital length of stay.

Goodness of fit for the logistic regression was assessed with the log likelihood ratio and the Wald and Hosmer-Lemeshow tests. For the proportional hazards Cox regression, we checked the proportionality of hazards by testing for non-zero slopes in a generalized linear regression of the scaled Schoenfeld residuals on functions of time, and additionally graphically by eye. A $p$ value of $<0.05$ was considered significant in all applied tests. As the type of procedure (that is, primary or revision) may affect the anesthesia type selection, an interaction term was included in the regression models but was removed later as it was not significant. SPSS software (version 21; IBM, Armonk, New York) was used for data handling, and R (version 2.15.3; Institute of Statistics and Mathematics, R Foundation of Statistical Computing) with the packages MatchIt and Survey was used for statistical analyses ${ }^{25,26}$.

\section{Source of Funding}

There was no external funding source for this study.

\section{Results}

Crom 2007 to 2011, 20,606 total hip arthroplasties were included in the NSQIP registry. Of these, 12,929 were from 
The Journal of Bone \& Joint Surgery - Jbjs.org Volume 97-A • Number $3 \cdot$ February 4, 2015
Effects of Regional Versus General Anesthesia on Outcomes After Total Hip arthroplasty

\section{TABLE I Characteristics of Patients Receiving Regional or General Anesthesia}

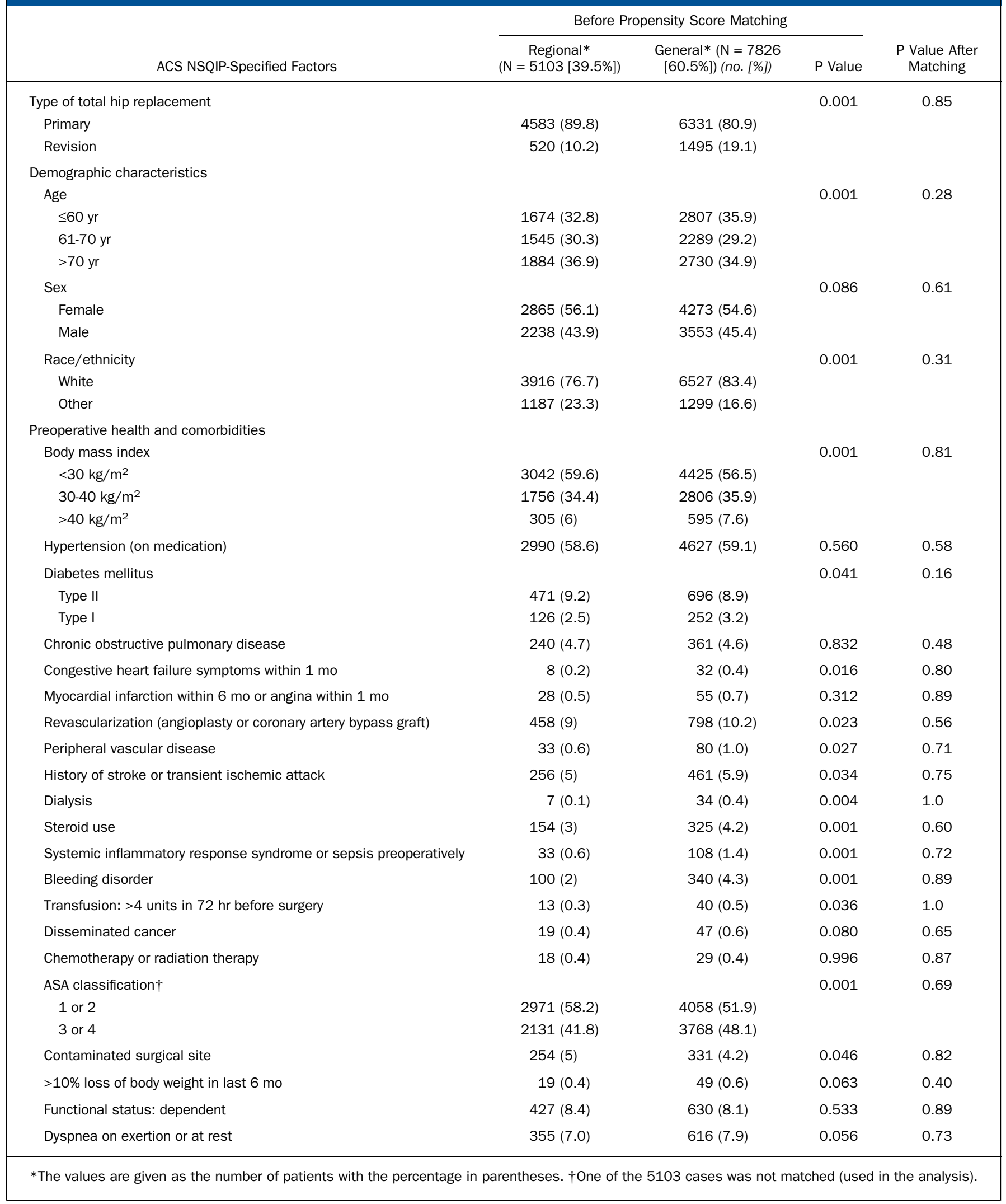




\section{TABLE II Unadjusted Outcomes of Regional Versus General Anesthesia Before and After Propensity Score Matching}

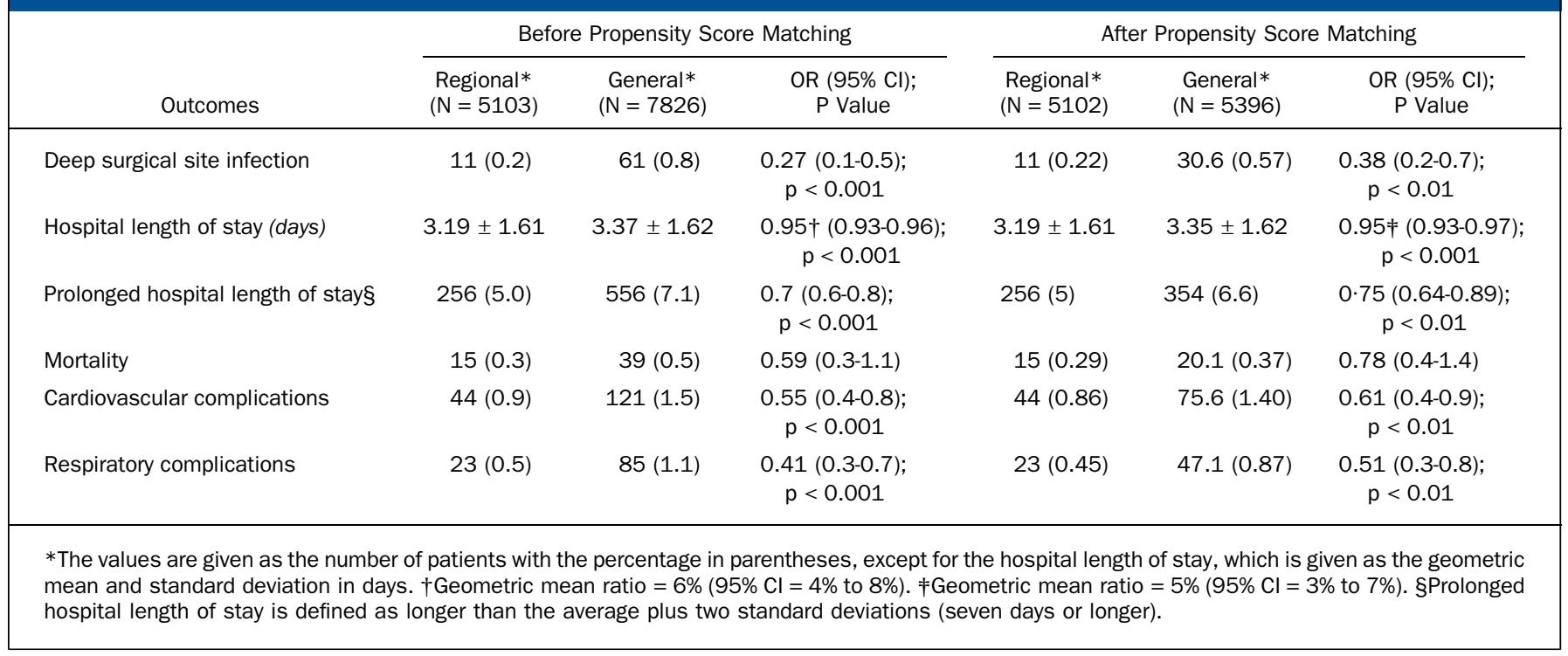

classic programs with no missing perioperative variables of interest (Fig. 1). There were 10,914 primary hip arthroplasties $(84.4 \%)$ and 2015 revision procedures (15.6\%). Regional anesthesia was used for $5103(39.5 \%)$ of the procedures, and general anesthesia was used for $7826(60.5 \%)$. The regional anesthesia was spinal in 4377 (85.8\%) of the cases, epidural in $126(2.5 \%)$, and "unspecified regional" in the remaining $600(11.8 \%)$. The rate of regional anesthesia in the non-classic programs was $39.1 \%$, and the rate of general anesthesia was $60.9 \%$.

Before propensity score matching, sixteen of the twentyfive baseline factors differed significantly between the two anesthesia groups. There were no significant differences between the groups after 1:2 matching and use of a caliper of 0.05 (Table I). All regional anesthesia cases except one (5102 cases) were matched to 5396 general anesthesia cases.

Deep surgical site infections were significantly less common after regional anesthesia than after general anesthesia $(0.22 \%$ compared with $0.57 \%)$, with an odds ratio (after matching) of 0.38 (95\% confidence interval $[\mathrm{CI}]=0.20$ to 0.72 ; $\mathrm{p}<0.01$ ). Deep surgical site infections were also associated with revision arthroplasties, age, and insulin-dependent diabetes (Tables II and III).

The unadjusted average hospital length of stay, after matching, was shorter after regional anesthesia (geometric mean and SD, $3.2 \pm 1.6$ days) than after general anesthesia (geometric mean, $3.4 \pm 1.6$ days). Proportionally, this yields an odds ratio (OR) for length of stay of 0.95 ( $95 \% \mathrm{CI}=0.93$ to 0.97 ) for regional anesthesia with a mean difference (compared with general anesthesia) of 0.05 ( $95 \% \mathrm{CI}=0.03$ to $0.07 ; \mathrm{p}<0.001)$, which translates to 0.17 day for each total hip arthroplasty (Table II).

The adjusted odds of the hospital length of stay were $8 \%$ lower in the regional anesthesia group $(\mathrm{OR}=0.92 ; 95 \% \mathrm{CI}=$ 0.89 to $0.94 ; \mathrm{p}<0.001$ ) (see Appendix). Figure 2 shows consistent earlier discharge (shorter hospital length of stay) in the regional anesthesia group compared with the general anesthesia group. The odds of a patient having a prolonged hospital length of stay, after we controlled for other factors, was $24 \%$ lower in the regional anesthesia group: 5\% compared with $6.6 \%$ in the general anesthesia group $(\mathrm{OR}=0.73 ; 95 \% \mathrm{CI}=$ 0.68 to $0.89 ; \mathrm{p}<0.001$ ).

Thirty-day mortality was a rare event, with an aftermatching prevalence of $0.29 \%$ in the regional anesthesia group and $0.37 \%$ in the general anesthesia group (Table II). In the multivariable analysis, the mortality odds were not significantly lower in the regional anesthesia group $(\mathrm{OR}=0.78 ; 95 \% \mathrm{CI}=0.43$

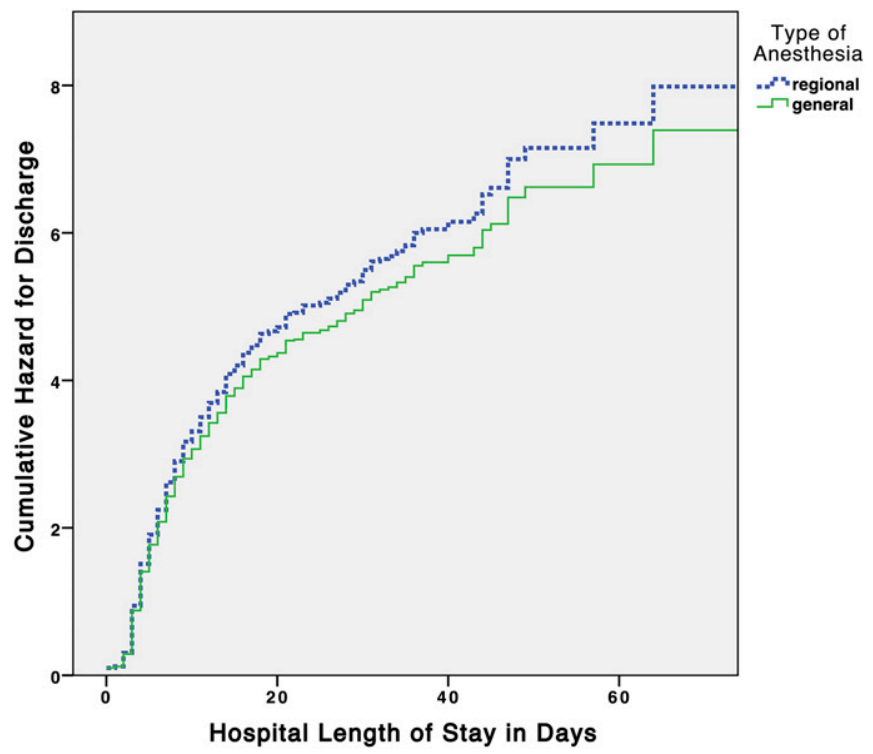

Fig. 2

Kaplan-Meier survival curves showing adjusted cumulative hazard of hospital discharge comparing regional anesthesia to general anesthesia. 
The Journal of Bone \& Joint Surgery - Jbjs.org Volume 97-A • Number $3 \cdot$ February 4, 2015
Effects of Regional Versus General Anesthesia on Outcomes After Total Hip Arthroplasty

TABLE III Results of Multivariable Logistic Regression Analyses for Deep Surgical Site Infection, Mortality, and Respiratory Complications

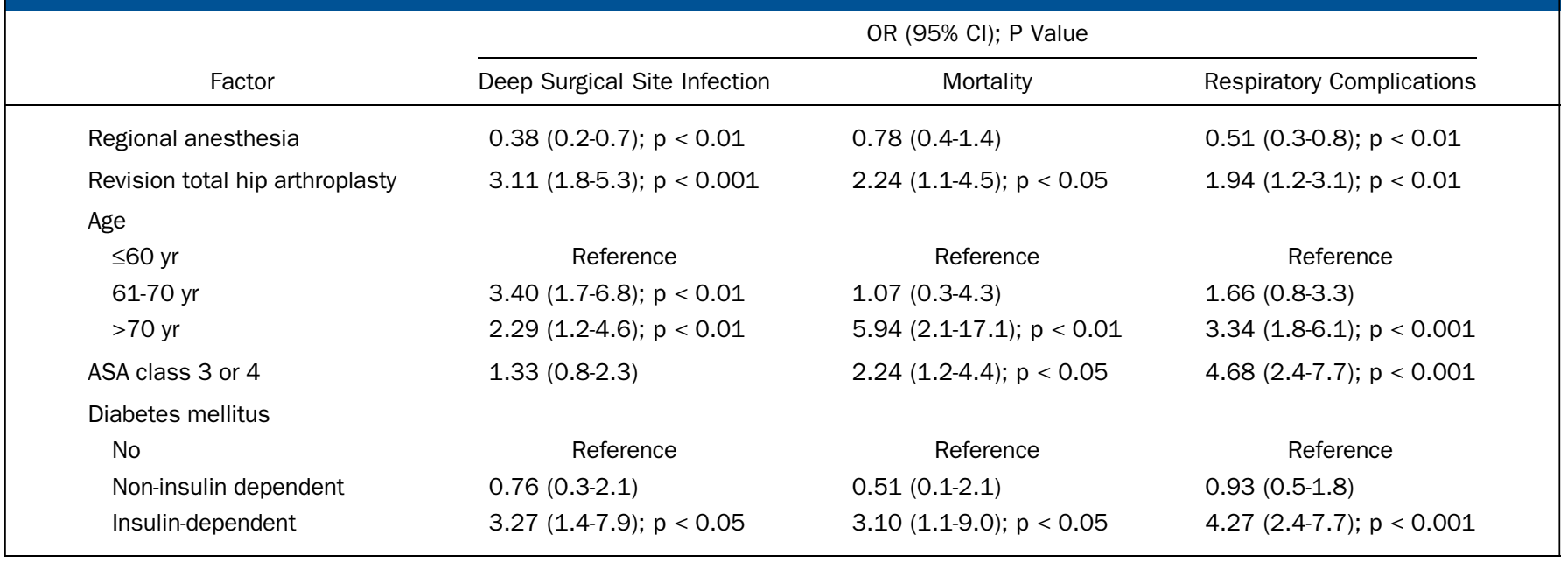

to $1.42 ; \mathrm{p}>0.05$ ) (Fig. 3 ). Factors that were significantly associated with increased postoperative mortality were revision total hip arthroplasty $(\mathrm{OR}=2.24 ; 95 \% \mathrm{CI}=1.12$ to $4.48 ; \mathrm{p}<0.05)$, an age of more than seventy years compared with an age of less than fifty years $(\mathrm{OR}=5.94 ; 95 \% \mathrm{CI}=2.06$ to $17.1 ; \mathrm{p}<0.01)$, ASA class
3 or 4 compared with ASA class 1 or $2(\mathrm{OR}=2.24 ; 95 \% \mathrm{CI}=1.15$ to 4.36; $\mathrm{p}<0.05)$, and insulin-dependent diabetes compared with no diabetes $(\mathrm{OR}=3.10 ; 95 \% \mathrm{CI}=1.1$ to $9.02 ; \mathrm{p}<0.05)$.

The adjusted odds for cardiovascular complications $(\mathrm{OR}=$ $0.61 ; 95 \% \mathrm{CI}=0.44$ to 0.85 ) and respiratory complications

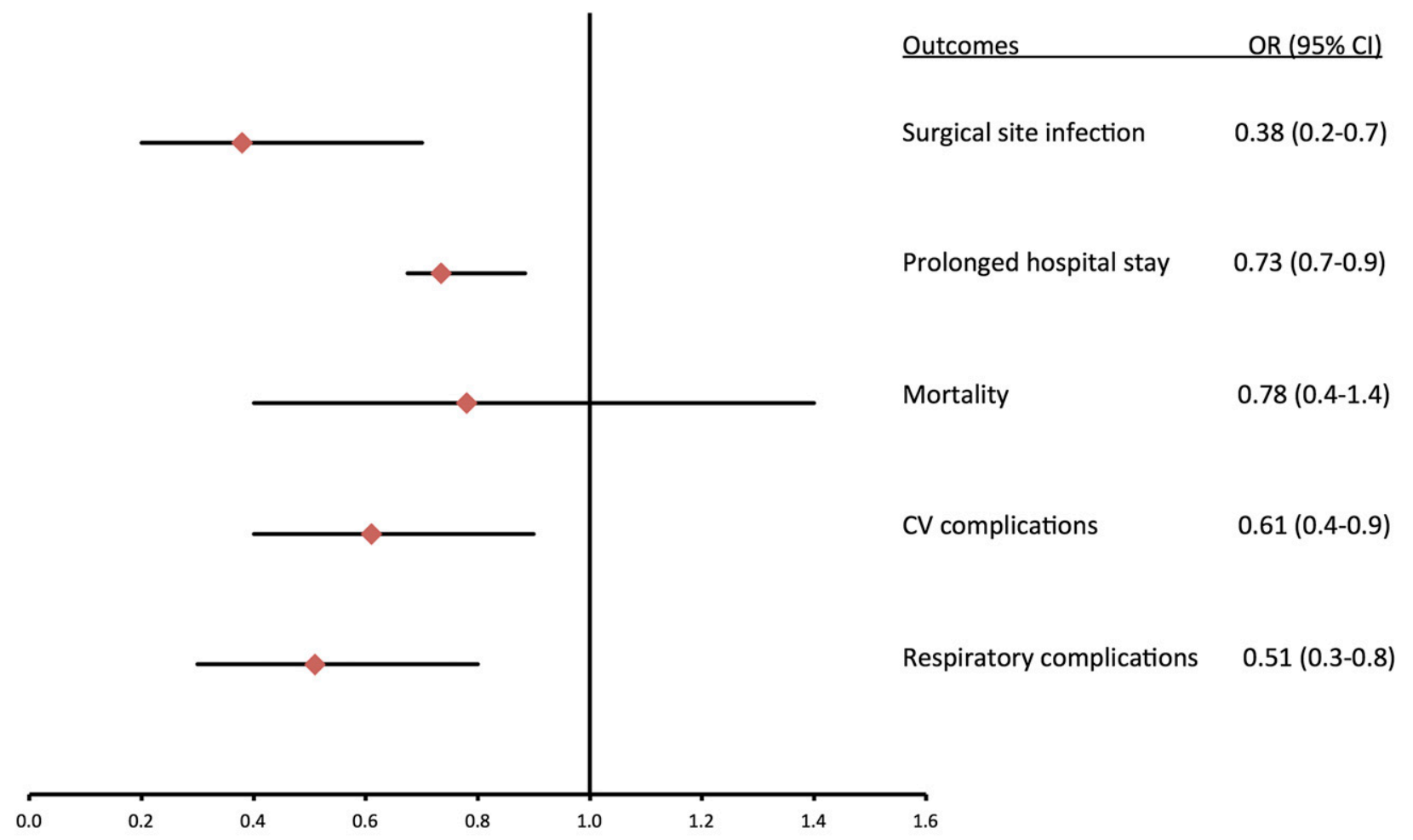

\section{$\mathrm{Cl}$ : Confidence Interval, CV: cardiovascular.}

Fig. 3

Logistic regression analysis result for outcomes comparing regional anesthesia with general anesthesia after propensity score matching. The diamonds indicate the odds ratios, which show a decreased odds for each outcome with regional anesthesia, and the lines through the diamonds indicate the $95 \% \mathrm{Cl}$. 
The Journal of Bone \& Joint Surgery $\cdot$ Jbjs.org Volume 97-A • Number $3 \cdot$ February 4, 2015
Effects of Regional Versus General Anesthesia on Outcomes After Total Hip Arthroplasty
$(\mathrm{OR}=0.51 ; 95 \% \mathrm{CI}=0.33$ to 0.81$)$ were all lower with regional anesthesia (Fig. 3).

Other important factors affecting the outcomes are summarized in Table III and a table in the Appendix.

\section{Discussion}

$\mathrm{U}$ sing the ACS NSQIP database and a propensity-matched analysis, we studied the impact of anesthesia type on thirtyday outcomes following total hip arthroplasty. Compared with general anesthesia, regional anesthesia was associated with impressive reductions in postoperative deep surgical site infection rates, hospital length of stay, and rates of cardiovascular and pulmonary complications. Regional anesthesia was associated with a nonsignificant $0.2 \%$ decrease in mortality. As mortality in the thirty days following total hip arthroplasty is rare, and the possible decrease in mortality attributable to regional anesthesia is low, a very large study would be needed in order to detect a mortality benefit attributable to regional anesthesia.

The severity of comorbidities as well as the duration of surgery, the length of the preoperative hospital stay, and low hospital volume have been identified as risk factors for deep surgical site infection following total hip replacement ${ }^{27}$. Preventive measures such as timely administration of antibiotics, maintenance of normothermia, and minimizing transfusions are well $\mathrm{known}^{28}$, but previous reports did not show regional anesthesia to have as important an effect on deep surgical site infections after total hip or knee replacement ${ }^{29,30}$. Possible mechanisms for how regional anesthesia lowers the prevalence of deep surgical site infection might be alterations in immune response $^{31}$ and sympathetic blocking effects with concomitant vasodilation improving tissue perfusion and oxygenation ${ }^{32}$.

Hospital length of stay is often used as a proxy for postoperative complications and resource utilization ${ }^{33}$. The study of prolonged hospital stay or length-of-stay outliers is essential for the management and financing of hospitals. We chose to define a prolonged length of stay as the geometric mean plus two standard deviations as this approach was superior to three others in predicting $\cos ^{34}$. Regional anesthesia was associated with decreases in all measures related to length of stay. Given that total hip arthroplasty is such a common surgical procedure, even a minor decrease in hospital length of stay could have major resource-savings implications.

Previous studies comparing regional anesthesia and general anesthesia have demonstrated conflicting results. Some studies have not shown benefits ${ }^{9,10}$, while others have demonstrated decreased blood loss, fewer respiratory complications, and a lower risk of thromboembolism with regional anesthe$\mathrm{sia}^{11,35,36}$. In a recent retrospective analysis from the National Joint Registry for England and Wales of ninety-day mortality after total hip replacement, spinal anesthesia, but not epidural anesthesia, was associated with a lower mortality rate ${ }^{5}$. The limitations of some of these studies as guides for current standards of care include relatively low patient numbers and changes in perioperative practice since the collection of the data. Memtsoudis and colleagues reported similar results concerning pulmonary, cardiac, and infectious complications in a large data set of patients who underwent total hip or knee arthroplasty under regional or general anesthesia ${ }^{37}$. That study had potential limitations; the database was administrative rather than medical, $27 \%$ of the cases had missing data on anesthesia type, a high percentage of patients received combined regional and general anesthesia, and the reported complication rates were in-hospital.

The credibility and general applicability of the main findings of our study are supported by several factors. First, the results are based on a recent, prospectively collected, multicenter data set of patients who underwent primary or revision total hip arthroplasty at a wide range of hospitals in both the private and the academic sector. Second, we utilized a propensity-score-matching technique in an attempt to balance factors affecting the exposure and regression models to control for confounders affecting the outcome. Third, the estimated prevalences of each of the individual complications included in the composite outcomes were consistently lower for patients who received regional anesthesia, suggesting benefit across complications. Fourth, the results are biologically plausible, as regional techniques and drugs block sympathetic nerve activity, reduce cytokine production, and inhibit platelet function ${ }^{7,8}$.

This study has important limitations. As it was not a randomized controlled trial, despite statistical corrections, unaccounted (unobserved) factors could have biased treatment exposure (to regional or general anesthesia), and hidden confounders might have biased the outcomes. For example, there could have been location bias, as the proportion of each anesthetic type might differ according to hospital center. Quality of care in general is likely to vary among hospital centers as well.

Another important limitation is that details about the general anesthetics were not considered. The outcomes associated with general anesthesia with long-acting agents, inadequate analgesia, hemodynamic shifts, and unnecessarily deep anesthesia might be very different from those associated with "fast-track" general anesthesia with rapid and predictable agents, multimodal analgesia, targeted hemodynamic control, and appropriately titrated anesthetic depth. These aspects and other unrecorded perioperative information (for example, postoperative pain and delirium) could have biased the outcomes.

In conclusion, although the ACS NSQIP utilizes a systematic sampling approach that is not guaranteed to provide a perfect estimate of procedure-type prevalence across the country, if the data observed here are reflective, then despite emerging evidence regional anesthesia appears to have been potentially underutilized during this study period (used for only $40 \%$ of total hip arthroplasties). The results reported here add credence to the notion that regional anesthesia is associated with a reduction in deep surgical site infection rates, length of hospital stay, and postoperative morbidity compared with general anesthesia. These findings, if replicated in appropriately designed effectiveness studies, could have an important medical and economic impact on health-care practice. 
The Journal of Bone \& Joint Surgery $\cdot$ Jbjs.org Volume 97-A · Number $3 \cdot$ February 4, 2015
Effects of Regional Versus General Anesthesia on Outcomes After Total Hip Arthroplasty

\section{Appendix}

eA A table showing the results of multivariable logistic revascular complications is available with the online version of this article as a data supplement at jbjs.org.

Mohammad A. Helwani, MD

Michael S. Avidan, MBBCh
Arbi Ben Abdallah, $\mathrm{PhD}$

Dagmar J. Kaiser, MD

John C. Clohisy, MD

Bruce L. Hall, MD

Heiko A. Kaiser, MD

Department of Anesthesiology (M.A.H., M.S.A., A.B.A., D.J.K., and H.A.K.), Department of Orthopedic Surgery (J.C.C.), and Department of Surgery (B.L.H.), Washington University in St. Louis School of Medicine, 660 South Euclid Avenue,

St. Louis, MO 63110.

E-mail address for M.A. Helwani: helwanim@wustl.edu

\section{References}

1. Kurtz $\mathrm{S}$, Ong $\mathrm{K}$, Lau E, Mowat F, Halpern M. Projections of primary and revision hip and knee arthroplasty in the United States from 2005 to 2030. J Bone Joint Surg Am. $2007 \mathrm{Apr} ; 89(4): 780-5$.

2. Fu KMG, Smith JS, Polly DW Jr, Ames CP, Berven SH, Perra JH, McCarthy RE, Knapp DR Jr, Shaffrey Cl; Scoliosis Research Society Morbidity and Mortality Committee. Correlation of higher preoperative American Society of Anesthesiology grade and increased morbidity and mortality rates in patients undergoing spine surgery. J Neurosurg Spine. 2011 Apr;14(4):470-4. Epub 2011 Feb 4.

3. Parvizi J, Johnson BG, Rowland C, Ereth MH, Lewallen DG. Thirty-day mortality after elective total hip arthroplasty. J Bone Joint Surg Am. 2001 Oct;83(10):1524-8. 4. Singh JA, Kundukulam J, Riddle DL, Strand V, Tugwell P. Early postoperative mortality following joint arthroplasty: a systematic review. J Rheumatol. 2011 Jul; 38(7):1507-13.

5. Hunt LP, Ben-Shlomo Y, Clark EM, Dieppe P, Judge A, MacGregor AJ, Tobias JH, Vernon K, Blom AW; National Joint Registry for England, Wales and Northern Ireland. 90-day mortality after 409,096 total hip replacements for osteoarthritis, from the National Joint Registry for England and Wales: a retrospective analysis. Lancet. 2013 Sep 28;382(9898):1097-104.

6. Kettner SC, Willschke $\mathrm{H}$, Marhofer $\mathrm{P}$. Does regional anaesthesia really improve outcome? Br J Anaesth. 2011 Dec;107(Suppl 1):i90-5.

7. Banz VM, Jakob SM, Inderbitzin D. Review article: improving outcome after major surgery: pathophysiological considerations. Anesth Analg. 2011 May;112(5):114755. Epub 2010 Aug 24.

8. Hollmann MW, Wieczorek KS, Smart M, Durieux ME. Epidural anesthesia prevents hypercoagulation in patients undergoing major orthopedic surgery. Reg Anesth Pain Med. 2001 May-Jun;26(3):215-22.

9. Neuman MD, Silber JH, Elkassabany NM, Ludwig JM, Fleisher LA. Comparative effectiveness of regional versus general anesthesia for hip fracture surgery in adults. Anesthesiology. 2012 Jul;117(1):72-92.

10. Le-Wendling L, Bihorac A, Baslanti TO, Lucas S, Sadasivan K, Wendling A, Heyman HJ, Boezaart A. Regional anesthesia as compared with general anesthesia for surgery in geriatric patients with hip fracture: does it decrease morbidity, mortality, and health care costs? Results of a single-centered study. Pain Med. 2012 Jul;13(7):948-56. Epub 2012 Jul 3.

11. Wijeysundera DN, Beattie WS, Austin PC, Hux JE, Laupacis A. Epidural anaesthesia and survival after intermediate-to-high risk non-cardiac surgery: a populationbased cohort study. Lancet. 2008 Aug 16;372(9638):562-9. Epub 2008 Aug 8. 12. Mauermann WJ, Shilling AM, Zuo Z. A comparison of neuraxial block versus general anesthesia for elective total hip replacement: a meta-analysis. Anesth Analg. 2006 Oct;103(4):1018-25.

13. Rodgers A, Walker N, Schug $S$, McKee A, Kehlet H, van Zundert A, Sage D, Futter M, Saville G, Clark T, MacMahon S. Reduction of postoperative mortality and morbidity with epidural or spinal anaesthesia: results from overview of randomised trials. BMJ. 2000 Dec 16;321(7275):1493.

14. O'Hara DA, Duff A, Berlin JA, Poses RM, Lawrence VA, Huber EC, Noveck H, Strom BL, Carson JL. The effect of anesthetic technique on postoperative outcomes in hip fracture repair. Anesthesiology. 2000 Apr;92(4):947-57.

15. Strum EM, Zelman V, Szenohradszki J, Reyes ML, McPherson EJ. [Primary and re-arthroplasty of the hip or knee joints in patients 80 years of age and older] [Russian]. Anesteziol Reanimatol. 2012 May-Jun;(3):4-8.

16. Memtsoudis SG, Sun X, Chiu YL, Stundner O, Liu SS, Banerjee S, Mazumdar M, Sharrock NE. Perioperative comparative effectiveness of anesthetic technique in orthopedic patients. Anesthesiology. 2013 May;118(5):1046-58.

17. von Elm E, Altman DG, Egger M, Pocock SJ, Gøtzsche PC, Vandenbroucke JP; STROBE Initiative. The Strengthening the Reporting of Observational Studies in Epidemiology (STROBE) statement: guidelines for reporting observational studies. J Clin Epidemiol. 2008 Apr;61(4):344-9.
18. Shiloach M, Frencher SK Jr, Steeger JE, Rowell KS, Bartzokis K, Tomeh MG, Richards KE, Ko CY, Hall BL. Toward robust information: data quality and inter-rater reliability in the American College of Surgeons National Surgical Quality Improvement Program. J Am Coll Surg. 2010 Jan;210(1):6-16. Epub 2009 Nov 22.

19. Austin PC. Some methods of propensity-score matching had superior performance to others: results of an empirical investigation and Monte Carlo simulations. Biom J. 2009 Feb;51(1):171-84.

20. Austin PC. Optimal caliper widths for propensity-score matching when estimating differences in means and differences in proportions in observational studies. Pharm Stat. 2011 Mar-Apr;10(2):150-61.

21. Stuart EA. Matching methods for causal inference: A review and a look forward. Stat Sci. 2010 Feb 1;25(1):1-21.

22. Bland JM, Altman DG. Transformations, means, and confidence intervals. BMJ. 1996 Apr 27;312(7038):1079.

23. Bland JM, Altman DG. The use of transformation when comparing two means. BMJ. 1996 May 4;312(7039):1153.

24. Singh JA, Jensen MR, Harmsen WS, Gabriel SE, Lewallen DG. Cardiac and thromboembolic complications and mortality in patients undergoing total hip and total knee arthroplasty. Ann Rheum Dis. 2011 Dec;70(12):2082-8. Epub 2011 Oct 21.

25. Ho DE, Imai K, King G, Stuart EA. Matching as nonparametric preprocessing for reducing model dependence in parametric causal inference. Polit Anal. 2007; 15: 199-236.

26. Lumley T. Analysis of complex survey samples. J Stat Softw. 2004;9(8):1-19. 27. Urquhart DM, Hanna FS, Brennan SL, Wluka AE, Leder K, Cameron PA, Graves $\mathrm{SE}$, Cicuttini FM. Incidence and risk factors for deep surgical site infection after primary total hip arthroplasty: a systematic review. J Arthroplasty. 2010 Dec;25 (8):1216-22.e1: 3. Epub 2009 Oct 30.

28. Sessler DI. Neuraxial anesthesia and surgical site infection. Anesthesiology. 2010 Aug;113(2):265-7.

29. Chang CC, Lin HC, Lin HW, Lin HC. Anesthetic management and surgical site infections in total hip or knee replacement: a population-based study. Anesthesiology. 2010 Aug;113(2):279-84.

30. Liu J, Ma C, Elkassabany N, Fleisher LA, Neuman MD. Neuraxial anesthesia decreases postoperative systemic infection risk compared with general anesthesia in knee arthroplasty. Anesth Analg. 2013 Oct;117(4):1010-6. Epub 2013 Sep 10.

31. Ciepichał J, Kübler A. Effect of general and regional anesthesia on some neutrophil functions. Arch Immunol Ther Exp (Warsz). 1998;46(3):183-92.

32. Treschan TA, Taguchi A, Ali SZ, Sharma N, Kabon B, Sessler DI, Kurz A. The effects of epidural and general anesthesia on tissue oxygenation. Anesth Analg. 2003 Jun;96(6):1553-7.

33. Cryer C, Gulliver P, Langley JD, Davie G. Is length of stay in hospital a stable proxy for injury severity? Inj Prev. 2010 Aug;16(4):254-60. Epub 2010 Jun 29.

34. Cots F, Elvira D, Castells $X$, Sáez M. Relevance of outlier cases in case mix systems and evaluation of trimming methods. Health Care Manag Sci. 2003 Feb; 6(1):27-35

35. Hu S, Zhang ZY, Hua YQ, Li J, Cai ZD. A comparison of regional and general anaesthesia for total replacement of the hip or knee: a meta-analysis. J Bone Joint Surg Br. 2009 Jul;91(7):935-42.

36. Park JH, Rasouli MR, Mortazavi SMJ, Tokarski AT, Maltenfort MG, Parvizi J. Predictors of perioperative blood loss in total joint arthroplasty. J Bone Joint Surg Am. 2013 Oct 2:95(19):1777-83.

37. Memtsoudis SG, Sun X, Chiu YL, Nurok M, Stundner O, Pastores SM, Mazumdar M. Utilization of critical care services among patients undergoing total hip and knee arthroplasty: epidemiology and risk factors. Anesthesiology. 2012 Jul;117(1): 107-16. 